\title{
Análisis de tres
}

\section{metodologías en la}

enseñanza del urbanismo

\author{
Analysis of three methodologies to teach \\ town planning
}

\section{Resumen}

Autores:

David Hidalgo García dhidalgo@ugr.es

Juan Manuel Santiago

Zaragoza

santi@ugr.es

Julián Arco Díaz

juliannn@ugr.es

Departamento de Expresión Gráfica Arquitectónica Universidad de Granada

España

Recibido: 25 Jun 2018 Aceptado: 29 Sep 2018 n los últimos años se han producido múltiples avances en relación A a la implantación de nuevas metodologías docentes dedicada a la impartición de asignaturas en el campo de la arquitectura y el urbanismo. Estas metodologías se encuentran avaladas por especialistas del ámbito de la pedagogía y la psicología, y su número de adeptos aumenta de forma considerable con el paso del tiempo. No obstante, dentro de estos campos es difícil encontrar estudios que consideren en profundidad la opinión de los agentes que intervienen en dichas metodologías, es decir: alumnado y profesorado. El objetivo de este artículo es conocer la opinión de estos agentes en la asignatura Proyecto de Desarrollo Urbano, impartida en la E.T.S. de Ingeniería de Edificación de la Universidad de Granada, tras la utilización de las tres metodologías más usadas en la docencia universitaria, y posteriormente, relacionar dichos datos con la mejora de sus competencias a través de los resultados obtenidos en su evaluación.

Palabras clave: Docencia universitaria, Flipped Classroom, Innovación Metodología y Proyectos.

\section{Abstract:}

Teaching methodologies of the subjects of architecture and urbanism have evolved in the late years along with the advances endorsed by specialists in pedagogy and psychology. The number of their followers is increasing. However, it is difficult to find, within these fields, studies that deepen taking into account the opinion of the agents that means the main role in these methodologies, i.e.: students and lecturers. The objective of this article is to know the opinion about the subject of Urban Development Project, lectured in the H.T.S. of Building Engineering of the University of Granada, using the three most important methodologies of university teaching: masterly lesson, flipped classroom and project-based learning. Later it should be related these data with the improvement of their competences through the results obtained through its evaluation.

Keywords: University teaching, Flipped Classroom, Innovation, Methodology and Projects. 


\section{Introducción}

Con la implantación del Espacio Europeo de Educación Superior se persiguen los siguientes objetivos: modernizar el sistema de educación superior, facilitar el intercambio de estudiantes y profesores europeos permitiendo la homologación de títulos entre los diferentes países, mejorar la calidad de la formación y el cambio en las denominaciones a: Grado, Máster y Doctorado. Este cambio supuso la conversión de los antiguos estudios universitarios, (Licenciaturas y Diplomaturas), en Grados, y con ello el establecimiento del Sistema Europeo de Transferencia de Créditos (ECTS) y la acreditación efectiva de la calidad de las titulaciones (Cuenca, 2013; Izquierdo, 2012) ${ }^{1}$. En los estudios conducentes a la profesión regulada de Arquitecto Técnico cabe indicar que se han transformado en un Grado de 4 años con un total de 240 créditos ECTS y la nueva denominación es la de Grado en Ingeniería de Edificación o Grado en Edificación.

Según el nuevo sistema, corresponde al profesorado de las Universidades la búsqueda e introducción de nuevas técnicas, metodologías o sistemas que mejoren el sistema de adquisición del conocimiento por parte del alumnado. Tal y como establece el Documento- Marco ${ }^{2}$ para la Integración del Sistema Universitario Español en el Espacio Europeo de Educación Superior:

La sociedad del conocimiento requiere innovaciones y cambios en las formas tradicionales de formación, producción, comunicación de la información y en el acceso a los servicios públicos y privados [...] Se hace pues, necesaria, una nueva concepción de la formación académica, centrada en el aprendizaje del alumno, y una revalorización de la función docente del profesorado universitario que incentive su motivación y que reconozca los esfuerzos encaminados a mejorar la calidad y la innovación educativa (Documento Marco, 2003, pp. 3-4).

El término metodología cuenta con múltiples definiciones. En líneas generales, puede ser definido como el camino a seguir para alcanzar uno o varios objetivos. El uso de las nuevas metodologías en los sistemas de enseñanza universitaria ha sido un tema habitual de discusión y debate durante la última década (Ontigueira,

1 Para saber más en relación a los cambios sufridos en el Grado en Edificación por la implantación del Espacio Europeo de Educación Superior, consultar: Izquierdo, P. (2012). Evolución histórica de los estudios, competencias y atribuciones de los Aparejadores y Arquitectos Técnicos. Madrid, España: Editorial Dyckinson, y Cuenca L.J. (2012). Aparejadores, Arquitectos Técnicos e Ingenieros de la Edificación: Una aproximación histórica a sus responsabilidades. Madrid, España: Editorial Dyckinson.

2 La información y documentación relativa al Espacio Europeo de Educación Superior de la Unión Europea puede consultarse en la web http://www.eees.es/es/home
2016), y no se puede negar que suponen un excelente camino a seguir por el profesorado universitario para alcanzar los objetivos de calidad establecidos en el Documento Marco reseñado (López, 2013). De esta forma, se posibilita el poder romper con un modelo de enseñanza tradicional y obsoleto basado en clases magistrales donde importa poco o nada el desarrollo del alumno que, habitualmente, presenta una actitud pasiva con respecto a su proceso de aprendizaje.

A esta línea del uso y posterior evaluación de las nuevas metodologías como sistema de mejora, se ha sumado el profesorado de la asignatura de Proyecto de Desarrollo Urbano del Grado en Edificación de la Escuela Técnica Superior de Ingeniería de Edificación de la Universidad de Granada.

En este sentido, el libro titulado: Innovación docente y buenas prácticas en la Universidad de Granada, establece que las tres metodologías más usadas en la docencia universitaria de esta institución son: Tradicional (60\%), Flipped Classroom (15\%), también conocida como Aula invertida, y Aprendizaje Basado en Proyectos (ABP) (25\%), (Jiménez del Barco, 2013).

La asignatura Proyecto de Desarrollo Urbano se imparte en el segundo semestre, (febrero- mayo), del tercer curso. Cuenta con una carga docente de 6 créditos ECTS, (3 Créditos teóricos y 3 créditos prácticos) y consta de 6 temas. Se distribuye en dos grupos: el $A$, en horario de mañana con 72 alumnos matriculados, y el grupo B, en horario de tarde con 42 alumnos. Ambos grupos son impartidos por un total de tres profesores.

Durante el curso académico 17-18, se acordó entre el profesorado de la asignatura, impartir dos temas con cada una de las metodologías establecidas $y$, posteriormente, distribuir entre el alumnado un cuestionario de satisfacción para evaluar su funcionamiento y las preferencias entre cada una de ellas. El objetivo del profesorado, con base en los resultados obtenidos, es implantar para el curso académico 18-19, la metodología docente seleccionada entre el alumnado.

De esta forma, y tras la evaluación final del alumnado de la asignatura, se han cruzado los datos obtenidos a través de las encuestas de opinión sobre la metodología, con los datos de rendimiento académico, observando que estos han resultado cuantitativamente superiores a los obtenidos en cursos anteriores donde solo se empleaba la metodología denominada como tradicional. Por tanto, no solo se trata de seleccionar la metodología más entretenida o divertida para el alumnado, sino la que además, permita obtener los mejores rendimientos académicos. 


\section{Materiales y métodos}

El ensayo comenzó en el momento en el que se desarrolló la presentación de la asignatura, en la primera clase del mes de febrero. Era fundamental que el alumnado estuviera informado de la metodología a seguir durante el proceso formativo y que formaban parte de una investigación, al objeto de que se convirtieran en un elemento activo y partícipe del proceso de aprendizaje a desarrollar con las diferentes metodologías propuestas.

En la tabla 1 se pueden observar las distintas fases en las que se dividió el proceso a desarrollar en este artículo.

\begin{tabular}{|c|l|l|}
\hline Fases & \multicolumn{1}{|c|}{ Desarrollo } & \multicolumn{1}{c|}{ Resultados } \\
Fase I & $\begin{array}{l}\text { Búsqueda y } \\
\text { selección de } \\
\text { Metodologías } \\
\text { más usadas en la } \\
\text { Universidad de } \\
\text { Granada }\end{array}$ & $\begin{array}{c}\text { - Tradicional } \\
\text { - Flipped Classroom } \\
\text { Fasendizaje } \\
\text { Basado en } \\
\text { Proyectos }\end{array}$ \\
\hline Fase III & $\begin{array}{l}\text { Diseño y } \\
\text { preparación de } \\
\text { recursos }\end{array}$ & $\begin{array}{l}\text { Materiales didácticos: } \\
\text { grabación de videos, } \\
\text { PowerPoint, etc. }\end{array}$ \\
\hline Fase IV el aula & $\begin{array}{l}\text { Valoración } \\
\text { alumnado }\end{array}$ & $\begin{array}{l}\text { Cambio en la } \\
\text { metodología de los } \\
\text { docentes }\end{array}$ \\
\hline Fase V & Conclusiones & $\begin{array}{l}\text { Resultados del } \\
\text { cuestionario y de la } \\
\text { evaluación }\end{array}$ \\
\hline
\end{tabular}

Tabla 1: Fases del Proceso llevado a cabo para el análisis de tres metodologías en la enseñanza del urbanismo. Fuente: Elaboración Propia.

El temario de la asignatura ha sido el siguiente:

\footnotetext{
- Tema 1: Introducción a la forma urbana.

- Tema 2: La ciudad antigua.

- Tema 3: La ciudad clásica.

- Tema 4: La ciudad medieval e islámica.

- Tema 5: La ciudad renacentista y barroca.

- Tema 6: La ciudad industrial y moderna.
}

Los temas 1 y 2 se desarrollaron con la metodología conocida como tradicional, es decir, el docente, durante el desarrollo de las clases, expone los contenidos de temario apoyándose en proyecciones con formato PowerPoint, (véase fig. 1).

Es necesario indicar que el estudio de la forma urbana a lo largo de la historia es una materia que habitualmente suele resultar bastante árida al alumnado de las carreras técnicas, acostumbrados a la resolución de un volumen importante de problemas matemáticos o constructivos. Esta circunstancia fue detectada por el profesorado de la asignatura algunos años atrás, lo que propició la elaboración de un material docente con presentaciones muy dinámicas y con un alto contenido audiovisual, basado en fotografías, películas, documentales, etc.

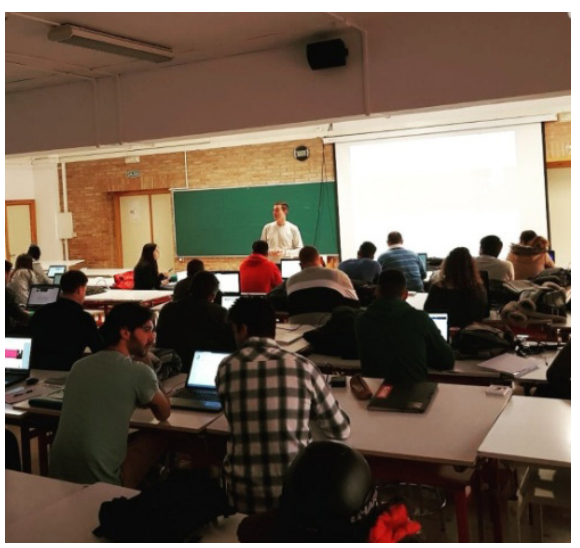

Figura 1: Impartición docente del profesor Dr. David Hidalgo en los Temas 1 y 2.

Fuente: Elaboración Propia.

Tal y como se observa en la figura número 2 , perteneciente al tema 2: La ciudad antigua, el contenido visual es superior al textual. En la parte inferior derecha existe un enlace a YouTube para visualizar un documental sobre las primeras civilizaciones. Estas presentaciones han permitido que la metodología, aun siendo tradicional, aumente la participación y creatividad del alumnado mediante la interacción entre estos y los recursos audiovisuales, transformando al alumno en un elemento activo de su proceso formativo.
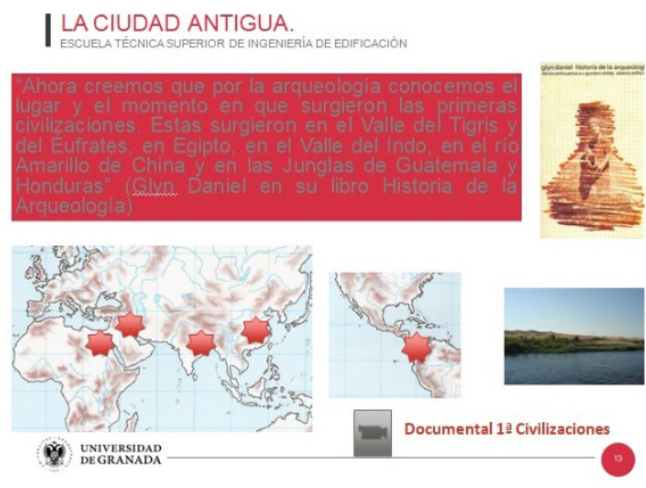

Figura 2: Diapositiva número 13 de la presentación de Tema 2: La ciudad antigua.

Fuente: Elaboración Propia.

Los temas 3 y 4 se desarrollaron con la metodología conocida como Flipped Classroom, o en castellano, aula invertida. Una metodología de reciente implantación que gana adeptos a ritmo considerable con el paso del tiempo ${ }^{3}$.

Consiste en un modelo pedagógico que invierte la metodología tradicional utilizada en la impartición de los

3 Consultar los estudios realizados por Prieto, A., Prieto, B. y De Pino, B. (2016). Una experiencia de flipped classroom. En Torres, M. y Cañadas, J. (Presidencia), XXII Jornadas sobre la Enseñanza Universitaria de la Informática. Almería, España. Los resultados de dicha experiencia fueron muy positivos, pasando el número de alumnos aprobados con el desarrollo de esta metodología del 57 a 
temas 1 y 2 , de tal forma que el alumno se prepara el tema fuera del horario de clase, destinándose estas a la resolución de ejercicios, prácticas o comentarios sobre artículos complementarios.

Este nuevo sistema de aprendizaje se remonta al año 2004, cuando los profesores de química de un instituto de Colorado (EE.UU.), Jon Bergmann y Aaron Sams, comienzan a grabar sus clases para posteriormente colgarlas en YouTube y que sirvieran como material de apoyo para los alumnos (Marqués, 2016).

Al ser la primera vez que se utilizaba esta metodología en la Escuela Técnica Superior de Ingeniería de Edificación, el profesorado de la asignatura, consciente de las posibles dificultades que podría tener el alumnado en casa, elaboró videos de corta duración con el contenido de los temas 3 y 4 . En concreto, un video por cada tema con una duración comprendida entre los 25 y 27 minutos, respectivamente. El proceso de elaboración fue mediante la inserción de audio a las presentaciones en formato PowerPoint. En la figura 3, se observa como en la parte inferior derecha de la diapositiva aparece el símbolo de audio.

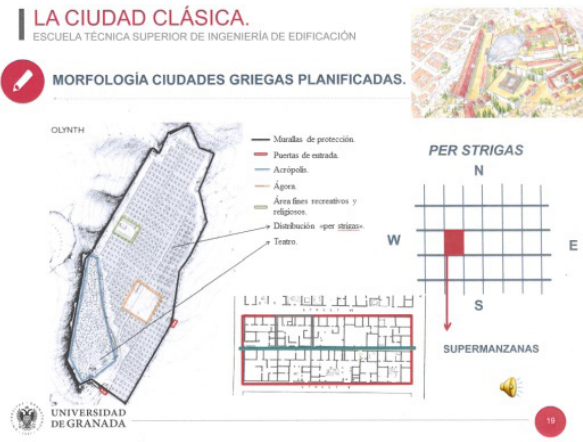

Figura 3: Diapositiva número 19 de la presentación con audio del Tema 3: La ciudad clásica.

Fuente: Elaboración Propia.

Tras la visualización de los temas por parte de alumnado fuera del horario de clase, el profesorado de la asignatura destinó el horario lectivo a comprobar, por un lado, la correcta adquisición de los conocimientos, y por otro, profundizar en los contenidos mediante la resolución de dudas, consultas, ejercicios y actividades complementarias que permitieran ampliar los conocimientos adquiridos por el alumnado mediante la visualización de los videos.

Los temas 5 y 6 se desarrollaron mediante el sistema de aprendizaje basado en proyectos (ABP). Un sistema novedoso que permite a los alumnos adquirir conocimientos y competencias mediante la resolución de proyectos reales en lugar de actividades teóricas. Mediante esta nueva metodología, el docente debe enfocar a los estudiantes en el camino adecuado para que adquieran las habilidades que necesitan en e futuro, tales como: comunicación, pensamiento crítico,

83\% e incrementándose la nota media de 5,7 a 6,3 puntos. colaboración y por supuesto, conocimiento (Soto, E., Santos, G., Pérez, S. y Mandado, E., 2018).

En palabras de los autores del artículo: Evaluación de la estrategia, aprendizaje basado en proyectos, el método $A B P$, consiste en:

Los estudiantes junto con los docentes persiguen soluciones a problemas no triviales, generando y refinando preguntas, debatiendo ideas, realizando predicciones, diseñando planes y/ o experimentos, recolectando y analizando datos, estableciendo conclusiones, comunicando sus ideas $y$ resultados a otros, realizando nuevas preguntas y creando o mejorando los procesos (Rodríguez, E., Vargas, E. y Luna, J, 2010, p.16)

El desarrollo de las clases mediante la metodología ABP supuso una mayor planificación por parte de los docentes en comparación con el resto de las metodologías empleadas.

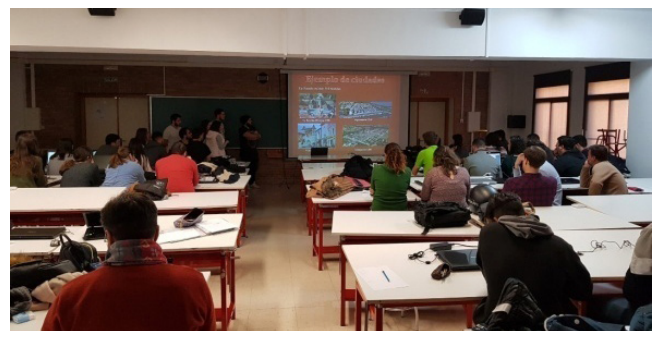

Figura 4: Exposiciones del tema 5: La ciudad renacentista y barroca por parte del alumnado.

Fuente: Elaboración Propia.

Esta dispuso de las siguientes fases:

\begin{tabular}{|l|l|}
\hline \multicolumn{1}{|c|}{ Fases } & \multicolumn{1}{c|}{ Desarrollo } \\
\hline Fase I & Subdivisión del alumnado en grupos de 4 \\
\hline Fase II & $\begin{array}{l}\text { Sorteo de los ítems del tema objeto de } \\
\text { estudio y consulta por cada grupo }\end{array}$ \\
\hline Fase III & $\begin{array}{l}\text { Tiempo de investigación tutelado por el } \\
\text { profesorado. Este, estableció al comienzo } \\
\text { de la clase unas cuestiones mínimas } \\
\text { a investigar en cada uno de los ítems } \\
\text { sorteados. Durante este periodo, el } \\
\text { profesorado resolvió las dudas generadas } \\
\text { en cada uno de los grupos, realizando una } \\
\text { labor de guiado hacia los objetivos finales } \\
\text { de cada ítem }\end{array}$ \\
\hline Fase IV & $\begin{array}{l}\text { Tiempo de preparación de la presentación } \\
\text { del ítem estudiado. }\end{array}$ \\
\hline Fase V & $\begin{array}{l}\text { Exposición pública del ítem al resto de } \\
\text { compañeros. }\end{array}$ \\
\hline Fase VI & $\begin{array}{l}\text { Debate abierto sobre dudas, consultas y } \\
\text { comentarios en relación a la exposición de } \\
\text { cada grupo }\end{array}$ \\
\hline
\end{tabular}

Tabla 2: Fases del desarrollo de clases con la metodología de Aprendizaje Basado en Proyectos (ABP).

Fuente: Elaboración Propia. 


\section{Resultados y Discusión}

Tras el desarrollo del temario establecido con las diferentes metodologías indicadas, se pidió al alumnado que completara un cuestionario en papel compuesto por siete preguntas y tres campos de observaciones. Cada metodología utilizada contaba con dos preguntas y un campo de observaciones. Cada pregunta se contestaba mediante una escala de tipo numérico cuyo intervalo oscila entre 0 y 10 , siendo el 0 un valor muy bajo y el 10 un valor muy alto, (véase fig.5).

Este cuestionario fue validado por otros profesores de la asignatura no participantes de la investigación mediante la técnica establecida por los autores Escobar-Pérez y Cuervo-Martínez, en 2008, denominada El juicio de expertos. Consiste en un método de validación adecuado para comprobar la viabilidad de una investigación. Esta, y en palabras de los autores indicados, consiste en:

Una opinión informada de personas con trayectoria en el tema, que son reconocidas por otros como expertos cualificados en este, y que pueden dar información evidencia, juicios y valoraciones (Escobar-Pérez, J. y CuervoMartínez, A., 2008, p.29).

El cuestionario se distribuyó en clase y la recogida se efectuó garantizando el anonimato de los encuestados.

El objetivo del cuestionario era el de conocer el grado de satisfacción del alumnado con cada una de las metodologías utilizadas, y determinar cuál consideraba más adecuada para su implantación en la asignatura de nuevo curso académico de la asignatura Proyecto de desarrollo urbano.

\section{ENCUESTA TEMARIO PROYECTO DE DESARROLLO URBANO.}

Durante la impartición de las temas de historia de la morfología urbana, se han usilizada diversas metodologias. Ios docentes queremos conocer la opirion del alumnado por cada una de ellas. Se toman como referencia lós sizguientes temas:

Temas 1 y 2: Introducción a la forma urbana y Cludad Antigua. Melodoloria: Habitual, es decir, explitationes por parte del docente.

Temas 3 y 4: Ciudad clásica y ciudad medieval e islámica. Metodolopia: Flipped Classroom

Temas 5 y 6: Ciudad Renacentista, Barroca y ciudad Industrial. Aprendizaje Basado en Propectos.

En base a ello, responder en base de 0-10, siendo 0 el muy bajo y 10 el muy alto.

1.- ¿Consideras adecuada la metodologia docente habitual uzilizada en la imparticion docente de los temas $1 \% 2$ ? Valore de 0 a $10:$

\begin{tabular}{|c|c|c|c|c|c|c|c|c|c|c|}
\hline 0 & 1 & 2 & 3 & 4 & 5 & 6 & 7 & 8 & 9 & 10 \\
\hline & & & & & & & & & & \\
2. - CConsideras
\end{tabular}

por su partecono excelenie?

te como excelente?

3.- ¿Cansideras adecuada la metodología docente Flipped Classroom utilizada en la impartición docente de ha temas 3 \% 4 ? Valore de 0 a 10

\begin{tabular}{|c|c|c|c|c|c|c|c|c|c|c|}
\hline 0 & 1 & 2 & 3 & 4 & 5 & 6 & 7 & 8 & 9 & 10 \\
\hline & & & & & & & & & & \\
\hline
\end{tabular}

por su parte como excelentu?

5.- ¿Consideras adecuada la metodologia Aprendizaje Basado en Proyectos (ABP) utilizada en

5.- ¿Consideras adecuada la metodologia Aprendizaje Basado

\begin{tabular}{|c|c|c|c|c|c|c|c|c|c|c|}
\hline $\mathbf{0}$ & $\mathbf{1}$ & $\mathbf{2}$ & $\mathbf{3}$ & $\mathbf{4}$ & $\mathbf{5}$ & $\mathbf{6}$ & $\mathbf{7}$ & $\mathbf{8}$ & $\mathbf{9}$ & 10 \\
\hline & & & & & & & & & & \\
\hline
\end{tabular}

por su parte como excelenze?

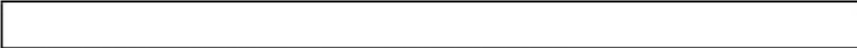

7.- ¿Por cuál de las tres te decantarias para la docercia recibida?

M. Habitual

M. Video+explicacioin $\square \quad$ M. irvertida $\square$
Mezcla de las tres $\square$

Figura 5: Cuestionario de opinión del alumnado sobre metodologías implantadas.

Fuente: Elaboración Propia. 


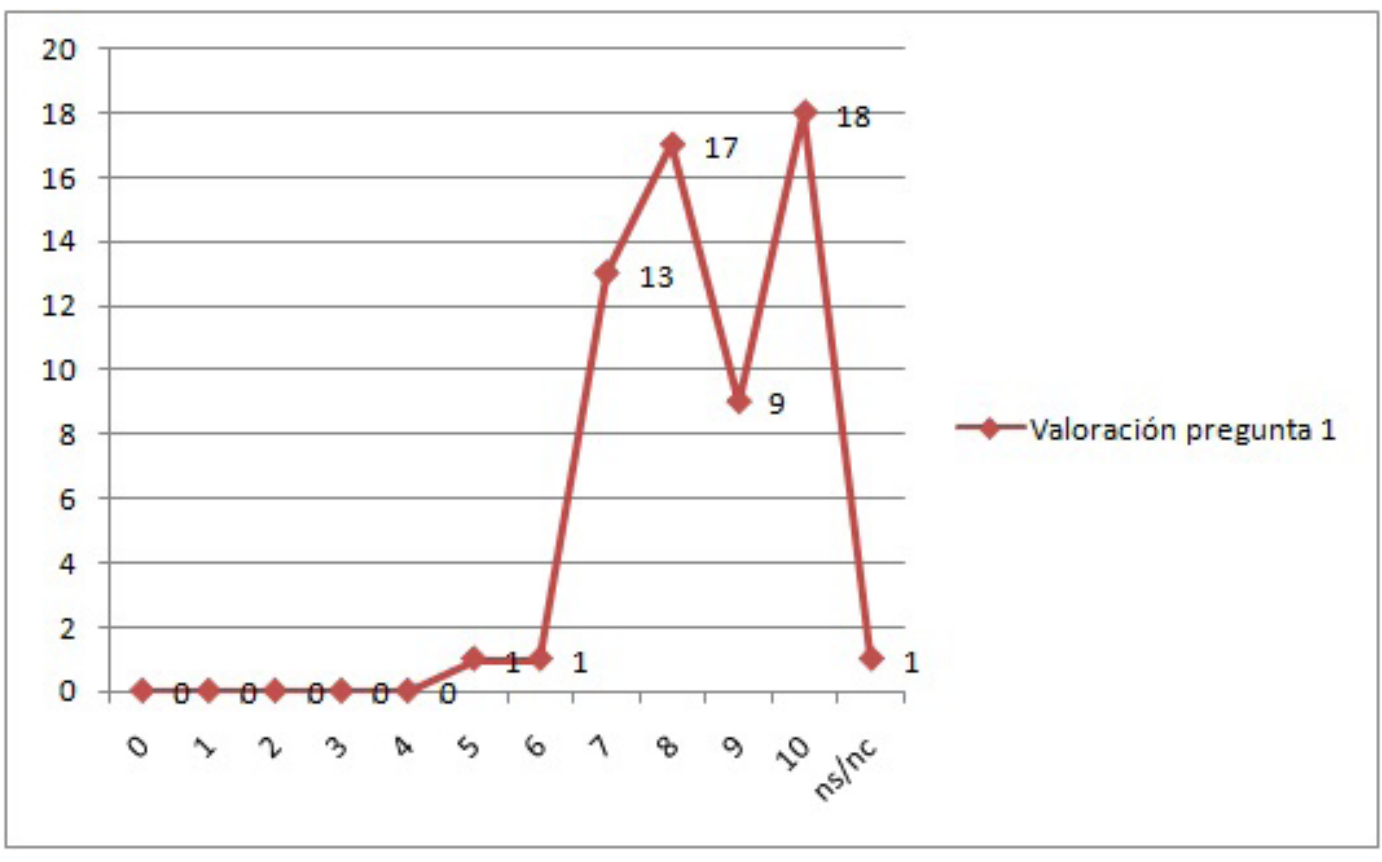

Figura 6: Resultados obtenidos en la pregunta 1 relacionada con la metodología tradicional. Fuente: Elaboración Propia.

Tras el estudio y análisis de los datos indicados por el alumnado en el cuestionario de satisfacción, los resultados por preguntas han sido los siguientes:

1) ¿Consideras adecuada la metodología docente habitual utilizada en la impartición de los temas 1 y 2 ? Valore de 0 a 10:

La pregunta fue contestada por 60 personas y obtuvo una valoración media de 8,46 puntos. En relación a la valoración otorgada por cada alumno, tal y como se observa en la figura 6, hubo 18 respuestas con un valor de 10. Es importante indicar que no hubo ninguna respuesta con una valoración inferior a 5 puntos. Estos datos son destacables, teniendo en cuenta que la metodología considerada como tradicional habitualmente se encuentra calificada como "obsoleta" y "desfasada" por un amplio abanico de autores (Aguilera, C., Manzano, A., Martínez, I., Lozano, M.C. y Casiano, C., 2017). En este caso, los datos demuestran que su valoración es bastante aceptable y contradicen las opiniones indicadas por estos autores.

En el campo de observaciones, el alumnado indicó como elementos de mejora para esta metodología, la necesaria introducción de más videos e imágenes en las presentaciones utilizadas durante la impartición docente. Una cuestión en la que coincidieron 6 alumnos.

2) ¿Consideras adecuada la metodología docente Flipped Classroom utilizada en la impartición de los temas 3 y 4 ? Valore de 0 a 10:

La pregunta fue contestada por 60 personas y obtuvo una valoración media de 8,48 puntos. Tan solo 2 centésimas por encima de la metodología tradicional. En relación a la valoración otorgada por cada alumno, tal y como se observa en la figura 7 , hubo 23 respuestas con un valor de 10. Sin embargo, hubo 1 respuesta con valor de 3 y un amplio número de respuestas (11 en total), con valores comprendidos entre 5 y 7 .

En el campo de observaciones de esta pregunta hubo mayor diversidad de respuestas. De esta forma, se valora en sentido positivo el poder visualizar los videos en cualquier momento y lugar y la calidad del material audiovisual. Por el contrario, y como elementos de mejora, establecen la necesidad de realizar videos de menor duración y la imposibilidad de resolver dudas durante la visualización de los videos. Esta cuestión es importante ya que el funcionamiento de la propia metodología establece que la resolución de las dudas originadas se desarrollará en la siguiente clase, pero el alumnado se siente vulnerable ante la imposibilidad de resolverlas en el momento en el que surgen.

3) ¿Consideras adecuada la metodología docente Aprendizaje Basado en Proyectos utilizada en la impartición de los temas 5 y 6 ? Valore de 0 a 10:

La pregunta fue contestada por 61 personas y obtuvo una valoración media de 7,00 puntos, un valor inferior en 1,46 y 1,48 puntos respectivamente en comparación con los datos obtenidos en las metodologías anteriores. En relación a la valoración otorgada por cada alumno, tal y como se observa en la figura 8 , hubo 10 respuestas con un valor de 10, sin embargo, hubo 1 respuesta con valor de 1 y un amplio número de respuestas, (29 en total), con valores comprendidos entre 5 y 7 . El valor más elegido, es decir, la moda, fue el de 7,00 puntos con 12 respuestas. 


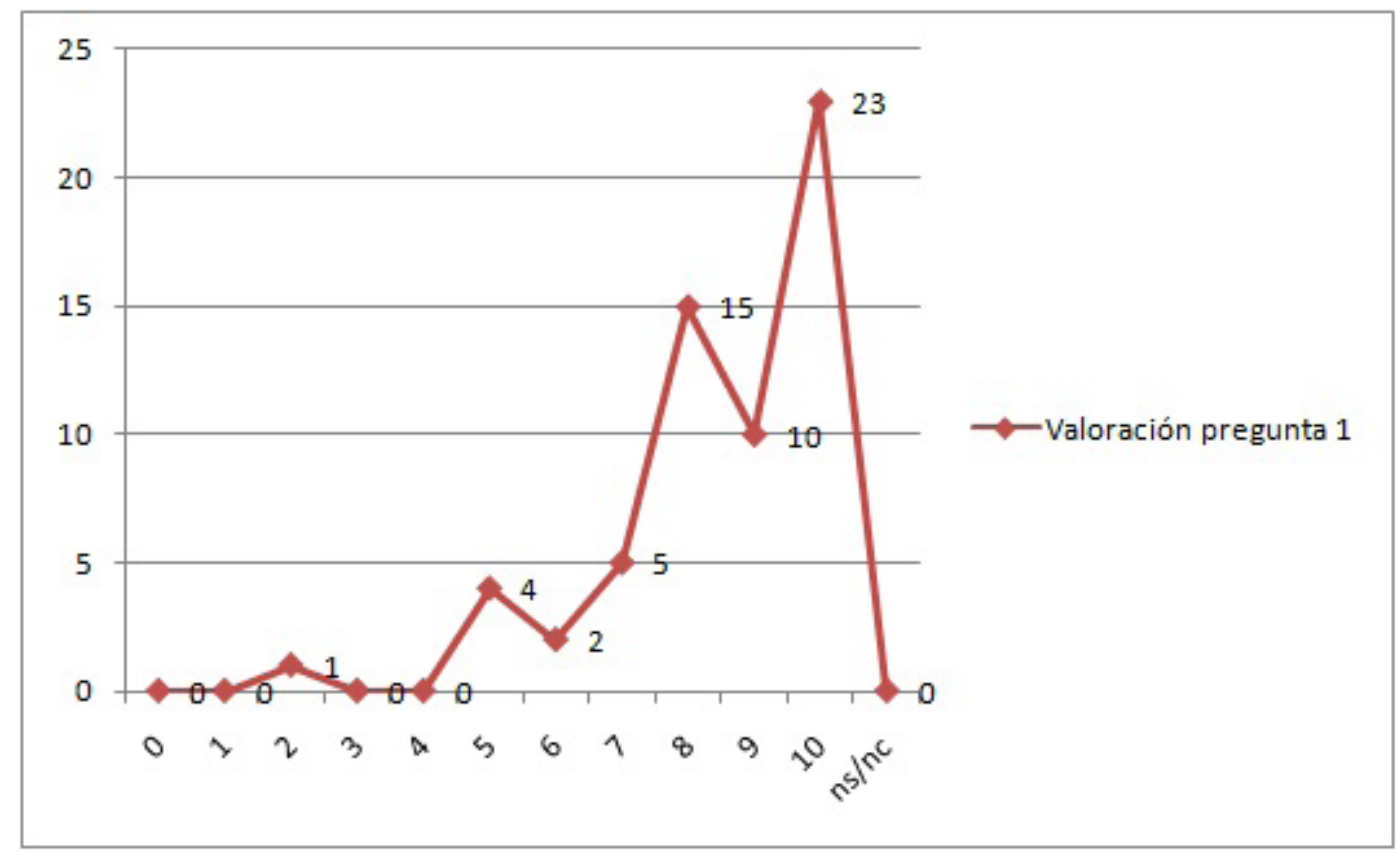

Figura 7: Resultados obtenidos pregunta 2 relacionada con la metodología Flipped Classroom. Fuente: Elaboración Propia.

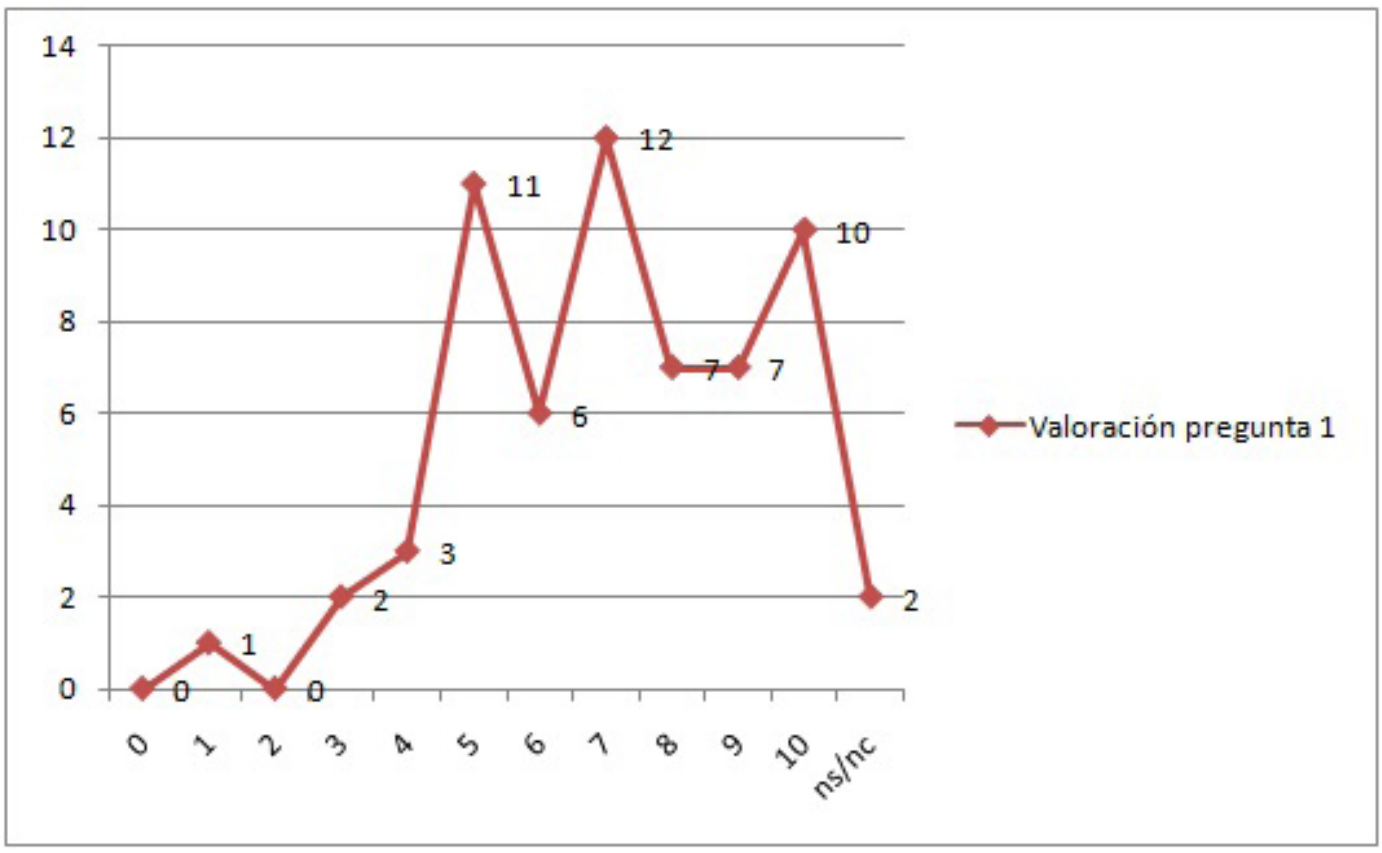

Figura 8: Resultados obtenidos pregunta 3 relacionada con la metodología Aprendizaje Basado en Proyectos. Fuente: Elaboración Propia. 


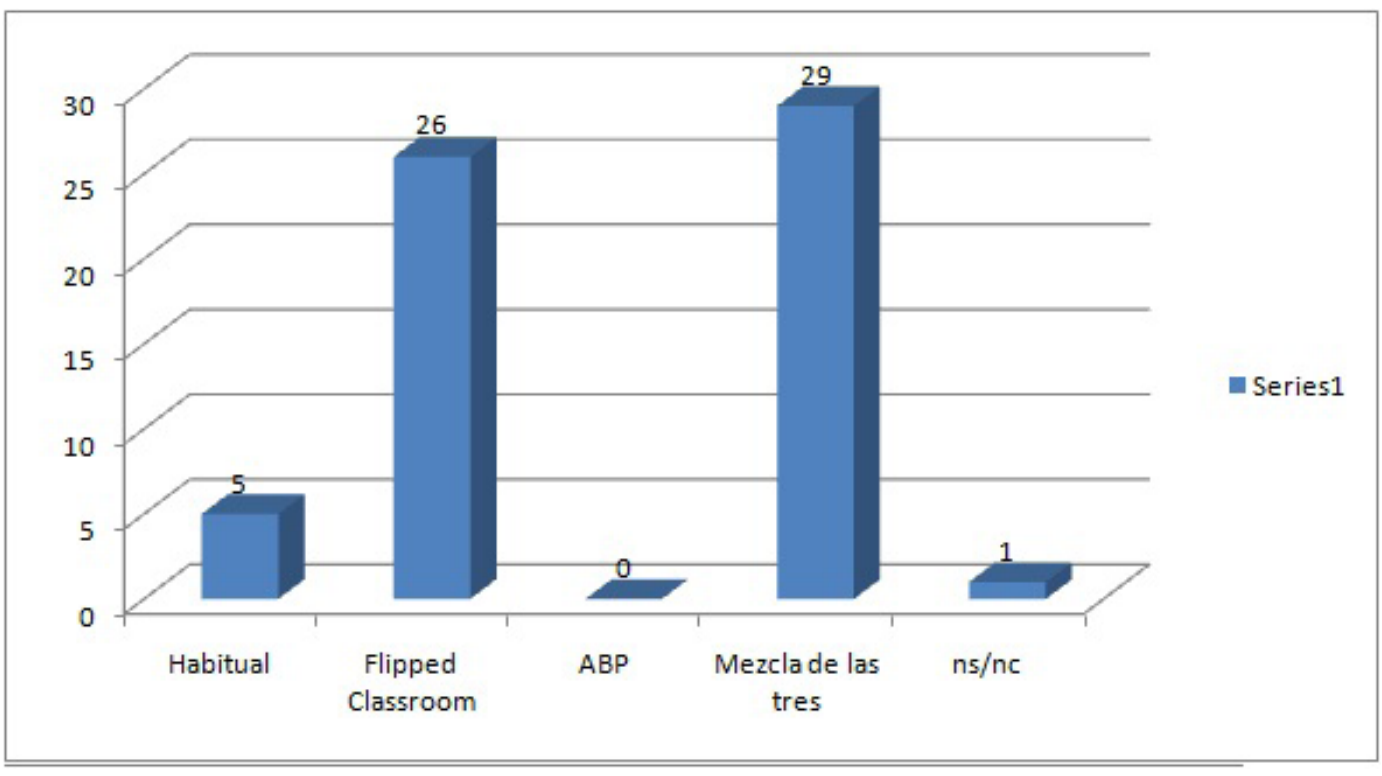

Figura 9: Resultados obtenidos pregunta 4 relacionada con la metodología más adecuada para la asignatura. Fuente: Elaboración Propia.

En el campo de observaciones no aparece ninguna referencia positiva a esta metodología. Por el contrario, todas negativas. En relación con estas últimas, el alumnado indicó como elementos de mejora la necesidad de disponer de mayor tiempo en la preparación de los temas, la realización de malas exposiciones por parte de algunos compañeros, y por último, la desconfianza que se genera entre el alumnado, aun estando el docente supervisando y guiando las presentaciones realizadas, por el contenido explicado por los compañeros. Son interesantes estos datos ya que contradicen las virtudes indicadas por algunos autores, (Soto et al., 2018; Marqués, 2016), en relación al uso de esta metodología.

Tras valorar cualitativamente las metodologías utilizadas en la impartición docente, se pidió al alumnado (pregunta 4 del cuestionario de la figura 5) que eligiera la que consideraba más adecuada para la impartición de la asignatura Proyecto de desarrollo urbano. Las respuestas obtenidas se pueden observar en la figura 9.

La pregunta fue contestada por 61 alumnos. De estos, 26 , el $42,5 \%$, eligieron la metodología denominada Flipped Classroom, 5 alumnos, el 8\%, la metodología conocida como tradicional y ninguno se decantó por la metodología ABP. Este último dato muy contrario con los datos positivos que diversos autores establecen sobre esta metodología (Sánchez, M., Solano, I. y González, V., 2016). Sin embargo, entre las posibles respuestas, se incluyó la posibilidad de no decantarse por una en concreto, sino elegir la alternancia de las 3 metodologías durante la impartición de los 6 temas. Esta última, fue la elegida mayoritariamente por el alumnado con 29 respuestas, es decir, que la alternancia en las metodologías docentes utilizadas era valorada por el $47,5 \%$ del alumnado como un elemento positivo y motivador para el correcto desarrollo de la impartición docente.
Esta alternancia de metodologías en los temas impartidos, elegida por el alumnado, ha sido la seguida por el profesorado de la asignatura para el desarrollo del ensayo descrito. Por tanto, el siguiente paso consiste en comprobar si esta alternancia ha tenido una repercusión directa sobre los resultados de evaluación académica del alumnado.

Durante el curso académico 16-17, la asignatura fue cursada por 114 alumnos. La metodología utilizada durante el desarrollo del temario fue la denominada como tradicional. Superaron la asignatura 72 alumnos lo que supone el $63 \%$ de los matriculados. La nota media del alumnado que superó la asignatura fue de 5,5 puntos, existiendo 2 alumnos con una nota de sobresaliente $y$ uno con matrícula de honor.

La evaluación obtenida durante el curso 17-18, con la alternancia en las metodologías docentes, arroja los siguientes datos: 82 alumnos han superado la asignatura, lo que supone el $73 \%$ de los matriculados; durante el curso 17-18 los matriculados han sido 112. La nota media del alumnado que ha superado la asignatura ha sido de 6,57 , existiendo 3 alumnos con matrícula de honor y ningún sobresaliente.

Con respecto a la opinión del profesorado de la asignatura en relación a la elección de una de las metodologías utilizadas, indicar que el mismo no se decanta por una en concreto. Todas conllevan una serie de elementos positivos y negativos con respecto al tiempo de preparación de clases, ejercicios, corrección de prácticas y preparación de material. Por tanto, los profesores y tras analizar los datos obtenidos en los cuestionarios de opinión del alumnado, son participes de seguir manteniendo la alternancia de metodologías para los próximos cursos académicos. 
Asimismo, cabe resaltar que a través de la alternancia de metodologías no solo se ha mejorado el proceso de adquisición de conocimiento por parte del alumnado, sino que también se observa en ellos una actitud más provechosa y receptiva, lo cual ha influido positivamente durante las clases.

\section{Conclusiones}

El uso de nuevas metodologías en la docencia universitaria ha sido objeto de estudio desde hace años. En la última década, y con el objetivo de romper con el desfasado sistema de enseñanza universitaria tradicional, junto con la necesidad de involucrar y hacer partícipe al alumno de su proceso de aprendizaje, se han puesto en marcha novedosas metodologías. Entre ellas se encuentran dos de las estudiadas en este artículo: Flipped Classroom y Aprendizaje Basado en Proyectos (ABP).

En líneas generales, los resultados obtenidos mediante la implantación de las tres metodologías didácticas han sido positivos, tanto para el profesorado como, principalmente, con el nivel de satisfacción y evaluación del alumnado. Resulta interesante como el alumnado de la asignatura Proyecto de desarrollo urbano no se decanta por una de ellas, sino que prefiere la alternancia de las tres como elemento motivador e innovador del proceso formativo, circunstancia que se refleja no solo en el número de alumnos que superan la asignatura, sino también en el incremento de la nota media del alumnado aprobado.

Resulta interesante cómo la metodología considerada "tradicional" y calificada de "obsoleta" obtiene una valoración media superior a 8 puntos, mientras que la novedosa metodología de Aprendizaje Basado en Proyectos, (ABP), se queda con una nota media de 7 puntos. Estas valoraciones contradicen las indicaciones de algunos autores citados anteriormente, lo que nos lleva a valorar estos resultados como preliminares, siendo necesario continuar con las investigaciones en los siguientes cursos académicos. También nos conduce a pensar si la enseñanza del urbanismo no sigue los mismos parámetros que la enseñanza de otras materias donde estas metodologías se encuentran contrastadas y con buenos resultados. Otras opciones a valorar podrían ser: la natural resistencia al cambio que ha podido experimentar el alumnado frente a esta nueva metodología (ABP), poco conocida por ellos en las clases que han recibido, un posible cambio de actitud del alumnado a lo largo del semestre, la mayor o menor empatía del alumnado con unos temas u otros, o el cambio en las relaciones entre profesorado y alumnado con el avance de las clases.

No obstante, el profesorado de la asignatura es crítico con su trabajo y con los datos obtenidos ya que, aunque son positivos, no debe olvidarse lo establecido por el autor Bloom (1984), cuando indica que el 90\% de los estudiantes se encuentran capacitados para adquirir los conocimientos y las competencias de las asignaturas. Por tanto, somos conscientes de que aún queda un largo camino por recorrer y mucho trabajo por realizar, si bien, como se deduce de los datos obtenidos en este artículo, la introducción de nuevas metodologías docentes nos acerca un poco más al objetivo marcado por el autor.

Como citar este artículo/How to cite this article: Hidalgo, D., Santiago, J.M. y Arco, J. (2018). Análisis de tres metodologías en la enseñanza del urbanismo. Estoa, Revista de la Facultad de Arquitectura y Urbanismo de la Universidad de Cuenca, 7(14), 7180. doi:10.18537/est.v007.n014.a05 


\section{Bibliografía}

- Aguilera, C., Manzano, A., Martínez, I., Lozano, M.C. y Casiano, C. (2016). El modelo Flipped Classroom. International Journal of Developmental and Educational Psychology, monográfico 3, (1), 261-266.

Bloom, B. (1984). The 2 Sigma Problem: The Search for Methods of Group Instruction as Effective as One- to One Tutoring. Educational Researcher, 13 (6), 4-16.

- Cuenca, L. J. (2013). Aparejadores, Arquitectos Técnicos e Ingenieros de la Edificación: una aproximación histórica a sus responsabilidades. Madrid, España: Editorial Dyckinson.

- Escobar-Pérez, J. y Cuervo-Martínez, A. (2008) Validez de contenido y juicio de expertos: una aproximación a su utilización. Avances en Medición, (6), 27-36. Recuperado de http:// www.humanas.unal.edu.co/psicometria/ files/7113/8574/5708/Articulo3_Juicio_de_ expertos_27-36.pdf

- Izquierdo, P. (2012). Evolución histórica de los estudios, competencias y atribuciones de los Aparejadores y Arquitectos Técnicos. Madrid, España: Editorial Dyckinson.

- Jiménez del Barco, L. (2013). Innovación docente y buenas prácticas en la Universidad de Granada. Granada, España: Editorial Universidad de Granada.

López, M.A. (2013). El uso de Twitter como herramienta para la enseñanza universitaria en el ámbito de las ciencias sociales. Un estudio de caso desde la historia económica. Teoría de la Educación: Educación y cultura en la Sociedad de la información, 14 (2), 316-345.
Marqués, M. (2016). Qué hay detrás de la clase al revés (flipped classroom). Revista de investigación en docencia universitaria de la informática. 9 (3), 11-18.

- Ministerio de Educación, Cultura y Deporte. (2003). Documento Marco para la integración del sistema universitario español en el Espacio Europeo de Educación Superior. Recuperado de http://tecnologiaedu.us.es/mec2011/htm/ mas/2/21/7.pdf

Ontigueira-Sánchez, L. (2016). Creación de habilidades y competencias a través del empleo de las nuevas tecnologías para el apoyo al proceso de aprendizaje. Revista Internacional de Gestión del Conocimiento y la Tecnología. 4, 39-45.

Rodríguez, E., Vargas, E. y Luna, J. (2010). Evaluación de la estrategia aprendizaje basado en proyectos. Educación y educadores. 13 (1), 13-25.

Sánchez, M., Solano, I. y González, V. (2016). FLIPPED-TIC: Una experiencia de Flipped Classroom con alumnos de Magisterio. Revista Latinoamericana de Tecnología Educativa. Volumen 15 (3), 69-81.

Soto, E., Santos, G., Pérez, S. y Mandado, E. (2018). El aprendizaje basado en problemas y proyectos combinado con el aprendizaje colaborativo en la enseñanza de la ingeniería. En A. Villa (Ed.), Tendencias actuales de las transformaciones de las universidades en una nueva sociedad digital, (pp. 209-216). Barcelona, España: Editorial Foro internacional de innovación universitaria. 